\title{
Sex-specific patterns of senescence in artificial insect populations varying in sex- ratio to manipulate reproductive effort
}

\author{
Charly Jehan*, Manon Chogne, Thierry Rigaud and Yannick Moret ${ }^{*}$ (i)
}

\begin{abstract}
Background: The disposable soma theory of ageing assumes that organisms optimally trade-off limited resources between reproduction and longevity to maximize fitness. Early reproduction should especially trade-off against late reproduction and longevity because of reduced investment into somatic protection, including immunity. Moreover, as optimal reproductive strategies of males and females differ, sexually dimorphic patterns of senescence may evolve. In particular, as males gain fitness through mating success, sexual competition should be a major factor accelerating male senescence. In a single experiment, we examined these possibilities by establishing artificial populations of the mealworm beetle, Tenebrio molitor, in which we manipulated the sex-ratio to generate variable levels of investment into reproductive effort and sexual competition in males and females.

Results: As predicted, variation in sex-ratio affected male and female reproductive efforts, with contrasted sexspecific trade-offs between lifetime reproduction, survival and immunity. High effort of reproduction accelerated mortality in females, without affecting immunity, but high early reproductive success was observed only in balanced sex-ratio condition. Male reproduction was costly on longevity and immunity, mainly because of their investment into copulations rather than in sexual competition.
\end{abstract}

Conclusions: Our results suggest that T. molitor males, like females, maximize fitness through enhanced longevity, partly explaining their comparable longevity.

Keywords: Ageing, Cost of reproduction, Disposable soma theory, Immunity, Immuno-senescence, Tenebrio molitor

\section{Background}

Life history theory assumes that organisms are constrained to optimally trade-off limited energetic and time resources between reproduction and life span, to maximize fitness $[1,2]$. This principle is at the core of the theory of ageing, which predicts that, as reproduction is resource demanding, current reproduction is traded-off against future reproduction and survival, caused by a reduced investment into somatic protection and maintenance [2-4]. However, recent studies have sometimes revealed patterns of actuarial (decline in survival rate with age) and reproductive (decline in reproductive success with age) senescence rather contrasted with this prediction [5-7]. Since individuals may differ in both resource

\footnotetext{
* Correspondence: charly.jehan@u-bourgogne.fr; yannick.moret@ubourgogne.fr

UMR CNRS 6282 BioGéoSciences, Équipe Écologie Évolutive, Université Bourgogne-Franche Comté, Dijon, France
}

acquisition and resource allocation between traits, depending on individual and environmental quality, the cost of reproduction can remain undetected at the population level $[8,9]$.

Studies that investigated cost of reproduction in terms of senescence mainly focused on females $[10,11]$. Those on males often referred to sexual selection theory and therefore on the cost of producing and maintaining sexual traits [12]. In males, cost of reproduction may result from resource demands for courtship, mating, struggling with female resistance, mate guarding, the production of sperm and accessory gland proteins [13-16]. They may also engage into costly intra-sexual competition for females through pre- and post-copulatory contests with other males [17]. In females, cost of reproduction may result from gamete production, offspring care, harassment by males, mating injuries, sexually transmitted diseases and damaging seminal substances [18-21]. These

(c) The Author(s). 2020 Open Access This article is distributed under the terms of the Creative Commons Attribution 4.0 International License (http://creativecommons.org/licenses/by/4.0/), which permits unrestricted use, distribution, and 
differential costs may have contributed to the evolution of sexually dimorphic life-history strategies in many species through which males and females achieve maximal fitness. For instance, while males may maximize fitness by increasing mating success at the expense of longevity, females may maximize fitness through longevity because offspring production, although resource intensive, requires time too. The different reproductive costs may also contribute to different patterns of senescence between males and females, which may vary within and among populations, depending on their relative intensity. Strong investment into reproduction early in life seems to contribute to accelerating reproductive and actuarial senescence [22]. However, our understanding of the impacts of the costs of reproduction on senescence mainly relies on theoretical and correlative studies, whereas experimental investigations are still scarce.

Somatic protection partly depends on the immune system, whose competence may diminish with age. Such an immunosenescence causes enhanced sensitivity to infection and inflammatory diseases, increasing risk of morbidity and mortality with age [23, 24]. Increased reproductive effort was found associated with enhanced susceptibility to parasitism and disease [25] or decreased immune activity [17, 26-29]. Trade-offs between reproductive and immune functions for limited resources, or negative pleiotropic effects of reproductive hormones on immune defence, have been proposed as proximate causes of the cost of reproduction [30]. However, contradictory results are common as studies also failed to demonstrate such a cost [31-33]. If investment into reproduction can induce a progressive decline in somatic functions, strong investment into early reproductive effort may generate accelerated immunosenescence and contribute to actuarial senescence.

Recent correlative evidence suggests that population structure, such as sex-ratio, affects individual reproductive effort with potential sex-specific consequences on senescence $[34,35]$. In particular, variation in sex-ratio is predicted to modulate the cost of mating, through the strength of sexual selection in males [36], influencing the putative trade-off between reproductive effort and somatic maintenance [11]. Furthermore, cost of reproduction in females is also predicted to depend on population sexratio as it is expected to influence male competition for fertilization [16]. Hence, experimentally varying population sex-ratio appears to be a valuable tool to manipulate males and females reproductive effort and test its impacts on senescence at the population level.

Here, in a single experiment, we investigated the consequences of variable levels of investment in breeding effort on lifetime reproduction, survival and immunity of males and females of the mealworm beetle, Tenebrio molitor, of which we have manipulated the sex-ratio in artificial populations. In this highly promiscuous insect, manipulating the sex-ratio of populations is expected to affect both the average intensity of intra sexual competition or sexual selection, and individual mating rate. In malebiased sex-ratio conditions, males should face fewer mating opportunities, whereas females should show high individual reproductive effort. By contrast, in female-biased sex-ratio condition, males should copulate more frequently, whereas females should have fewer opportunities to mate. This experimental design allowed us to test the cost of different key features of male and female reproduction in terms of senescence at the population level by examining their lifetime changes in survival, fertility, reproductive effort, body condition and immunity. Note, however, that manipulating sex-ratio may only affect the opportunity for sexual selection and not the actual sexual selection [37], and lifehistory particularities of biological models should be taken into account. For example, common wisdom is that malebiased sex-ratio conditions should accelerate male reduction of survival, reproduction and immunity because of intense pre- and post-copulatory intra sexual competition. However, in T. molitor, mating might be where the largest costs arise in reproduction for males (see below), and accelerated senescence is expected in populations with femalebiased sex-ratio because males should produce higher reproductive effort. Indeed, direct observations of the mating behaviour of T. molitor suggest that males do not engage in costly physical contests to access females [38, 39]. Courtship and mating are relatively brief during which males transfer a spermatophore that does not release the sperm before $7-10 \mathrm{~min}$ post-copulation [40]. Males may then perform rather passive short postcopulatory mate guarding, consisting on staying within $1 \mathrm{~cm}$ of the female for more than.

One minute in the presence of competitors [38, 39]. However, males do not appear to have evolved specific post-copulatory mate-guarding behaviour like those observed in other insects [41]. The spermatophore transferred during copulation contains nutrient-rich substances that constitute a nuptial gift [42], whose cost may prevent males to copulate again for $20 \mathrm{~min}$ after the last copulation [41]. Hence, as mating is more costly than pre- and post-copulatory sexual competition, T. molitor males may best achieve fitness through longevity, just like females, which would ultimately prevent the evolution of divergent patterns of actuarial senescence between males and females. Females, for their part, may exhibit strong early reproductive effort in populations with male-biased sexratio, they also should exhibit accelerated decline in reproduction, and immunity or earlier immune dysregulation, correlating with reduced survival with age. In populations with female-biased sex-ratio, females should survive, reproduce and maintain immunity at older age, as they might exhibit lower early life reproductive effort. 


\section{Methods}

\section{Mealworm beetles}

Mealworm beetles are stored grain product pests that live several months in populations of variable density and at sex-ratio of about 50\% ( $\pm 20 \%)$. T. molitor males and females may initiate reproduction from the fifth day post emergence, although they reach their full sexual maturity from the eighth day post emergence. They can mate many times with several partners within their 2 to 5 months of adult life. Females are continuously receptive to mating during adulthood and may produce up to 30 eggs per day although egg production may decline after 3 weeks [43]. Although able to store sperm in their spermatheca, females need to mate frequently to maintain high egg production [44].

The immune system of $T$. molitor relies on both constitutive cellular (e.g. haemocytes) and enzymatic (e.g. prophenoloxidase system) components at the core of the inflammatory response [45]. Their activity is cytotoxic [46], causing self-damage [47] and lifespan reduction [48-51]. They were found to decrease after mating [52] and either decline [53] or increase [54] with age. In addition, the inducible production of antibacterial peptides in the haemolymph [45] is an energetically costly process that may reduce survival [55]. As selection on immune expression and immune regulation might be weaker after reproductive senescence, age-related decline of baseline levels of immunity might be observed and immune activation may occur at old age due to dysregulation $[54,56]$.

\section{Artificial populations and experimental design}

Virgin adult beetles of controlled age ( $10 \pm 2$ days postemergence) were obtained from pupae haphazardly sampled from a stock culture maintained in laboratory conditions $\left(24 \pm 2{ }^{\circ} \mathrm{C}, 70 \% \mathrm{RH}\right.$ in permanent darkness) at Dijon, France. Prior to the experiments, all these experimental insects were maintained separately in laboratory conditions, and supplied ad libitum with bran flour and water, supplemented by apple.

Fifteen artificial populations of 100 adult beetles were made according to three sex-ratio conditions. Five populations had a balanced sex-ratio, each comprising 50 males and 50 females (thereafter named the 50\%_males condition), and were considered as the reference populations. Five populations had a male-biased sex-ratio, each comprising 75 males and 25 females (75\%_males). Finally, five populations had a female-biased sex-ratio, each comprising 25 males and 75 females (25\%_males). Each population was maintained in a plastic tank $(\mathrm{L} \times 1$ $\mathrm{x} \mathrm{H}, 27 \times 16.5 \times 11.5 \mathrm{~cm}$ ) containing bran flour, supplied once a week with apple and water. Every 2 weeks, each population was transferred into a clean tank supplied with fresh bran flour, thus avoiding the development of the progeny with the experimental adults.

\section{Age specific reproductive assay}

Reproductive capacity of females and males in each population was estimated weekly. To this purpose, 4 females haphazardly picked in each population were individually transferred into a plastic Petri dish $(9 \mathrm{~cm}$ in diameter), containing bleached flour, a $2 \mathrm{~mL}$ centrifuge tube of water and a piece of apple. Each female was allowed to lay eggs in the Petri dish for 3 days, and was then returned to their initial population box. Two weeks later, the number of larvae was counted in each Petri dish to quantify female fertility, which is the number of viable larvae produced per female [57].

Concomitantly, four males haphazardly picked in each population were also individually transferred into Petri dishes, as above. Reproductive success of males was estimated through direct measures of their fertility (number of viable offspring per male [57]) instead of measuring spermatophores or counting the sperm, which are rough surrogates of male reproductive success. Each male was provided with a virgin female aged from 8 to 15 days for $24 \mathrm{~h}$ and was then returned in its initial population. Each female was then allowed to lay eggs in the Petri dish for three additional days to estimate, as described above, male fertility. In $T$. molitor, males may affect female fecundity (number of potential eggs produced by the female) and therefore their fertility, according to the respective quality of spermatophores and sperm transferred during mating. Consequently, male's success was a measure of the potential reproductive effort, not the one realized within its experimental population.

While assayed for their reproduction, focal females and males were replaced by marked individuals of the same age and sex in all the populations, to keep sexratio and density constant. Substitutes were from the same cohort as the experimental insects, kept in a separate tank of mixed-sex population. They were marked by clipping a piece of one elytra. When focal insects assayed for their reproduction were returned into their initial population box, substitutes were removed and returned into their tank.

\section{Estimation of male and female reproductive effort at the population level}

Survival of the insects was checked weekly, and dead insects were replaced by marked substitutes of the same sex and about the same age to keep the population sexratio and density of individuals constant. No measurement was performed on these marked individuals.

As the experimental design does not allow gathering measurements of longevity and fertility for each individual of the population, we estimated male and female reproductive effort (RE) at the population level, from the above age-specific measures of fertility, for each of the five population replicates, within sex-ratio conditions. 
This estimate was calculated as the total number of viable larvae produced per female or male in each replicate (i.e. the cumulative number of larvae produced during the whole experiment in a given replicate divided by the number of females or males tested for this replicate), divided by their respective average lifespan in the population replicate (i.e. the average lifetime of females or males in each relicate). The equation is given as follow:

$$
R E_{r}=\frac{l}{M L}
$$

Where $l$ is the total number of offspring (here viable larvae) produced per assayed females or males in the population replicate $r$, and $M L$ is the recorded mean lifespan (in weeks) of males and females in the replicate $r$. $\mathrm{RE}$ values (as offspring per individuals and per mean weeks of survival in the population) of each sex and population replicate within each sex-ratio condition were used as data points for comparisons between modalities of sex-ratio.

Note that female RE values are likely representative of both female and male conditions resulting from the experiment, because the female reproductive performance resulted from mating with males from their respective population. By contrast, male RE values are representative of the male condition only, because male reproductive performance was standardized by pairing it with a virgin and age-controlled female that did not experience the experimental conditions. Therefore, male RE must be seen as a surrogate of male reproduction potential.

\section{Body condition and haemolymph collection}

At weeks 2, 4, 6 and 12 after the start of the experiment, 4 females and 4 males were picked at random in each population to estimate their body condition and immunity. The first three time points were chosen as being relevant of the time period during which most of the beetle reproduction is achieved and survival is still relatively high [44]. It is also within this period of time that a potential decline in somatic protection, including immunity, is predicted. The last time point corresponds to a period of time when reproduction should have almost ceased and when few beetles remain alive. As immunity measurements was destructive sampling, sampled insects were replaced by marked substitutes as above, to keep sex-ratio and density constant. However, this substitution was definitive, as sampled individuals were not returned to their initial population box after being assayed. The below estimation of the insect body condition and immunity was done as described in [58]. Beetles were first sized by measuring the length of the right elytra with Mitutoyo digital callipers (precision $0.1 \mathrm{~mm}$ ) and weighed to the nearest $\mathrm{mg}$ with an OHAUS balance (discovery series, DU114C). Body condition was then estimated by the residuals of the regression between body size and body mass. Then, beetles were chilled on ice for 10 min before the sampling of $5 \mu \mathrm{L}$ of haemolymph from a wound made in the beetle's neck and flushed in a microcentrifuge tube containing $25 \mu \mathrm{L}$ of phosphatebuffered saline (PBS $10 \mathrm{mM}, \mathrm{pH} 7.4$ ). A $10-\mu \mathrm{L}$ aliquot was immediately used to measure haemocyte count. Another $5-\mu \mathrm{L}$ aliquot was kept in an $\mathrm{N}$-phenylthioureacoated microcentrifuge tube (P7629, Sigma-Aldrich, St Louis, MO, USA) and stored at $-80^{\circ} \mathrm{C}$ for later examination of its antibacterial activity. The remaining haemolymph solution $(15 \mu \mathrm{L})$ was further diluted in $15 \mu \mathrm{L}$ of PBS and stored at $-80^{\circ} \mathrm{C}$ for later measurement of its phenoloxidase activity.

\section{Immune parameters}

Haemocyte count was measured using a Neubauer improved haemocytometer under a phase-contrast microscope (magnification $\times 400$ ).

Antimicrobial activity of the haemolymph was measured using the inhibition zone assay described in [58]. Briefly, an overnight culture of the bacterium Arthrobacter globiformis from the Pasteur Institute (CIP105365) was added to a Broth medium containing $1 \%$ agar to achieve a final concentration of $10^{5}$ cells. $\mathrm{mL}^{-1}$. Six millilitres of the medium was subsequently poured per Petri dish and, after solidification, 12 wells were made inside the agar plate in which $2 \mu \mathrm{L}$ of each haemolymph sample was deposited. Plates were then incubated overnight at $28^{\circ} \mathrm{C}$ and the diameter of each zone of inhibition was measured.

For each haemolymph sample, both (i) the activity of naturally activated phenoloxidase (PO) enzyme only (hereafter PO activity) and (ii) the activity of PO plus that of proenzymes (proPO) (hereafter Total-PO activity) were measured using the spectrophotometric assay described in [59]. Total-PO activity quantification required the activation of proPO into PO with chymotrypsin, whereas PO activity was measured directly from the sample. Frozen haemolymph samples were thawed on ice and centrifuged $\left(3500 \mathrm{~g}, 5 \mathrm{~min}, 4{ }^{\circ} \mathrm{C}\right)$. In a 96-well plate, $5 \mu \mathrm{L}$ of supernatant were diluted in $20 \mu \mathrm{L}$ of PBS and were added either $140 \mu \mathrm{L}$ of distilled water to measure PO activity or $140 \mu \mathrm{L}$ of $0.07 \mathrm{mg}$. $\mathrm{mL}^{-1}$ chymotrypsin solution (Sigma-Aldrich, St Louis, MO, USA, C7762) to measure Total-PO activity. Subsequently, $20 \mu \mathrm{L}$ of a $4 \mathrm{mg} \cdot \mathrm{mL}^{-1}$ L-Dopa solution (Sigma-Aldrich, St Louis, MO, USA, D-9628) were added to each well. The reaction proceeded for $40 \mathrm{~min}$ at $30^{\circ} \mathrm{C}$, in a microplate reader (Versamax, Molecular Devices, Sunnyval, CA, USA). Reads were taken every $15 \mathrm{~s}$ at $490 \mathrm{~nm}$ and analysed using the software SOFT-Max ${ }^{\circ}$ Pro 4.0 (Molecular 
Devices, Sunnyvale, CA, USA). Enzymatic activity was measured as the slope (Vmax value: change in absorbance unit per min) of the reaction curve during the linear phase of the reaction and reported to the activity of $1 \mu \mathrm{L}$ of pure haemolymph.

\section{Statistics}

Cox-regressions with a time-dependent covariate were used to analyse the difference in survival rates with respect to sex-ratio during the time (in weeks) from the start of the experiment and the death of all individuals. Sex-ratio was coded as categorical variables. The effect of sex ratio in the statistical model used the reference survival function generated from the data derived from the females or the males of the $50 \%$-male sex-ratio condition. Time (in weeks) was incremented as a covariate in interaction with sex-ratio in the model as hazard ratios when the survival functions where not constant over time (for more details, see [60]). The analyses of fertility (i.e. the number of larvae produced per female or male at each week) and immune parameters were performed using mixed models, either Linear or Generalized linear depending on the nature of the data (see table legends). Starting models included sex-ratio condition, week (continuous variable for fertility, ordinal variable for immunity), their interaction, body condition and replicates treated as a random factor (REML estimates of variance component). The models presented here are those minimizing the AICc, where $\triangle \mathrm{AICc}>2$ is usually considered to be good support [61], after comparisons of all models including predictors and their interactions, in a stepwise fashion (see Additional file 1: Table S1). The analyses of reproductive effort were made using ANOVA testing the effect of sex-ratio conditions. Analyses were made using $\mathrm{IBM}^{\circ} \mathrm{SPSS}^{\circ}$ Statistics 19, JMP v. 10.0 and R version 3.3.2 (The R Foundation for Statistical Computing, Vienna, Austria, http://www.r-project.org). All the data files are available from the Dryad data base [62].

\section{Results}

\section{Demography: survival, fertility and reproductive effort}

A first survival analysis comparing males and females of the 50\%-male sex-ratio condition, which presumably corresponds to the sex-ratio condition in natural populations of T. molitor, showed no difference in longevity between males and females (Cox regression: Wald statistics $=0.004$, d.f. $=1, p=0.947$, see Additional file 1 : Figure S1). Survival of females and males was significantly affected by the sex-ratio condition (Table 1, Fig. 1). In the 75\%-male sex-ratio condition, females exhibited an accelerated mortality with time by a factor of $13 \%$ per week compared to females of the 25 and 50\%-male sexratio conditions (see odd ratio of Sex-ratio*Time-Cov in Table 1a). There was no significant difference in survival between females in the 25 and 50\%-male sex-ratio conditions (Table 1a, Fig. 1a). Contrasting with females, males in the $75 \%$-male sex-ratio condition survived significantly longer than those in the 25 and 50\%-male sex-ratio conditions (by 53 and $50 \%$, respectively, see odd ratio in Table 1b, Fig. 1b).

Whereas female fertility decreased with time, this pattern was dependent on the sex-ratio condition (Table $2 \mathrm{a}$, Fig. 2a, see Additional file 1: Figure S2). Indeed, female fertility in the 75 and 25\%-male sex-ratio conditions was lower than that in the 50\%-male sex-ratio condition during the first 2 weeks, and became subsequently higher

Table 1 Survival of adult females (a) and males (b) of Tenebrio molitor according to sex-ratio condition (Sex-ratio). The "simple" contrast was used for Sex-ratio (survival of males in the $50 \%$ of male condition was used as baseline). For females (a), a timedependent procedure was used to account for the time-dependent effect of Sex-ratio on the risk of mortality ( $T \times$ Sex-ratio). This procedure was not necessary for males as the effect of Sex-ratio on the risk of mortality was constant over time (b)

\begin{tabular}{|c|c|c|c|c|c|c|}
\hline A. Variables & $b^{a}$ & s.e. $^{\mathrm{b}}$ & Wald $^{c}$ & d.f. & $p^{d}$ & Odds ratio ${ }^{\circ}$ \\
\hline Sex-ratio & & & 1.61 & 2 & 0.744 & \\
\hline$T \times$ Sex-ratio & & & 9.77 & 2 & 0.008 & \\
\hline $75 \%$ of males vs $50 \%$ of males & 0.12 & 0.04 & 9.28 & 1 & 0.002 & 1.13 \\
\hline $25 \%$ of males vs $50 \%$ of males & 0.00 & 0.02 & 0.05 & 1 & 0.831 & 1.00 \\
\hline $75 \%$ of males vs $25 \%$ of males & 0.12 & 0.04 & 9.03 & 1 & 0.003 & 1.12 \\
\hline B. Variables & $b$ & s.e. & Wald & d.f. & $p$ & Odds ratio \\
\hline Sex-ratio & & & 21.34 & 2 & $<0.001$ & \\
\hline $75 \%$ of males vs $50 \%$ of males & -0.38 & 0.10 & 15.36 & 1 & $<0.001$ & 0.70 \\
\hline $25 \%$ of males vs $50 \%$ of males & 0.15 & 0.17 & 0.82 & 1 & 0.366 & 1.16 \\
\hline $75 \%$ of males vs $25 \%$ of males & -0.53 & 0.16 & 10.98 & 1 & 0.001 & 0.59 \\
\hline
\end{tabular}

${ }^{\mathrm{a}} b=$ regression coefficient of overall survival function for variables

${ }^{\text {b }}$ Standard error of regression coefficient

'Wald statistic for variable

${ }^{\mathrm{d}}$ Significant level for Wald statistic. Values $p \leq 0.005$ are given in bold

eOdds ratio of survival for variable relative to control (=exp.(b)) 

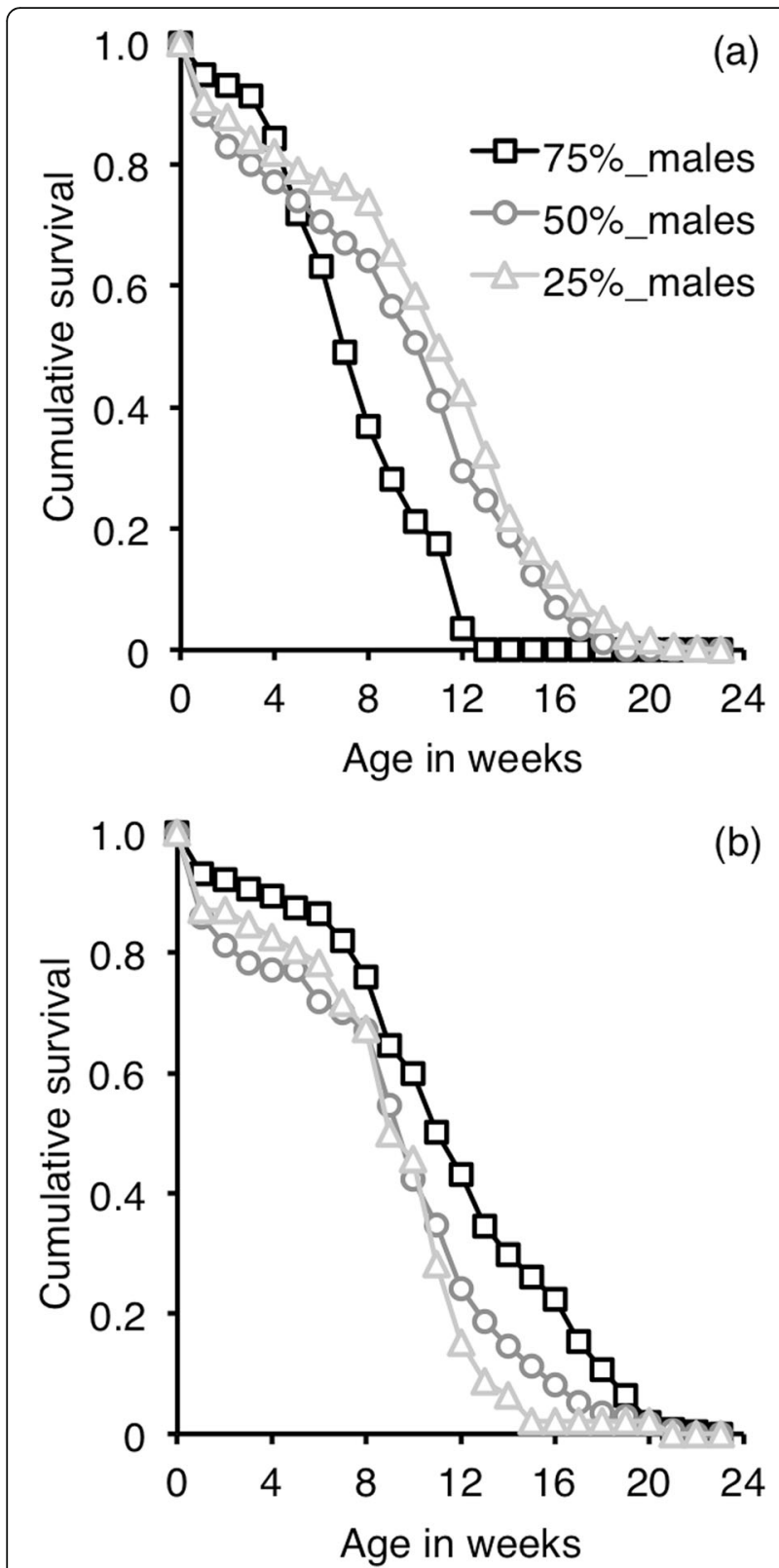

Fig. 1 Age-specific survival according to sex-ratio condition. a females; $\mathbf{b}$ males
(Fig. 2a). Male fertility decreased with time in all sex-ratio conditions, with no significant effect of sex-ratio condition (Table 2b, Fig. 2b, see Additional file 1: Figure S2). As expected for both sexes, heavier females produced more larvae than lighter ones (Table 2).

Female's RE differed among sex-ratio conditions $\left(F_{2}, 12=8.06 p=0.006\right)$ and was significantly higher in the $75 \%$-male than in the $25 \%$-male sex-ratio condition (Fig. 3a). Female RE in the 50\%-male sex-ratio condition showed an intermediate value (Fig. 3a). Male RE significantly differed among sex-ratio conditions $\left(F_{2,12}=4.63 p=0.032\right)$. Male $\mathrm{RE}$ in the $75 \%$ male sex-ratio condition was significantly lower than in the 25\%-male sex-ratio condition (Fig. 3b). Like for females, male's RE from the balanced sex-ratio condition showed an intermediate value, which was not significantly different from the two other sexratio conditions (Fig. 3b).

\section{Body condition and immunity}

Male and female body condition, estimated by the residuals of the regression between body size and body mass, exhibited a similar decline with age, which was not affected by the sex-ratio condition (Table 3a, see Additional file 1: Figure S3). In females, immunological parameters were never affected by the sex-ratio condition (Table 3b-f). Both PO activity and Total-PO activity changed with age with lowest values at week 6 (Fig. 4a), and were positively influenced by body condition (Table $3 \mathrm{~b}, \mathrm{c}$ ). By contrast, anti-bacterial activity of the haemolymph increased with age (Table 3e, Fig. 4b). Haemocyte counts of females only differed among population replicates (Table 3d, see Additional file 1: Figure S3). As opposed to females, some of the immunological parameters of males were affected by the sex-ratio condition (Table 3). Male PO activity was influenced by the interaction between time and sex-ratio (Table $3 \mathrm{~b}$ ). While PO activity of males in the 50\%-male sex-ratio condition decreased during the first 6 weeks, PO activity of males in the other two sex-ratio conditions increased between week 2 and 4 . In all sex-ratio conditions, PO activity increased

Table 2 Fertility: generalized linear mixt models (GLMM, Poisson distribution, Log link function) analysing the factors influencing the number of larvae produced by females (a), $(n=638)$ and males (b), $(n=737)$

\begin{tabular}{|c|c|c|c|c|c|c|c|}
\hline \multicolumn{4}{|l|}{ A } & \multicolumn{4}{|l|}{ B } \\
\hline Sources & d.f. & $x^{2}$ & $p$ & Sources & d.f. & $x^{2}$ & $p$ \\
\hline Female mass & 1 & 82.755 & $<0.001$ & Female mass & 1 & 243.13 & $<0.001$ \\
\hline Age & 1 & 388.9 & $<0.001$ & Age & 1 & 94.819 & $<0.001$ \\
\hline Sex-ratio & 2 & 0.7232 & 0.277 & & & & \\
\hline Sex-ratio $x$ Age & 2 & 100.44 & $<0.001$ & & & & \\
\hline
\end{tabular}

Initial models included Sex-ratio condition, Age (in weeks as a continuous variable), their interaction, male and female body mass (Mass in mg) and replicates as a random factor. The models shown here are those minimizing the AICc, with $\delta \mathrm{AICC}>2$. Age was allowed to vary up to 11 and 13 weeks in females and males, respectively. Beyond these values no insects could be assayed anymore in some sex-ratio conditions (see Additional file 1: Figure S2). Values where $p \leq 0.05$ are given in bold 

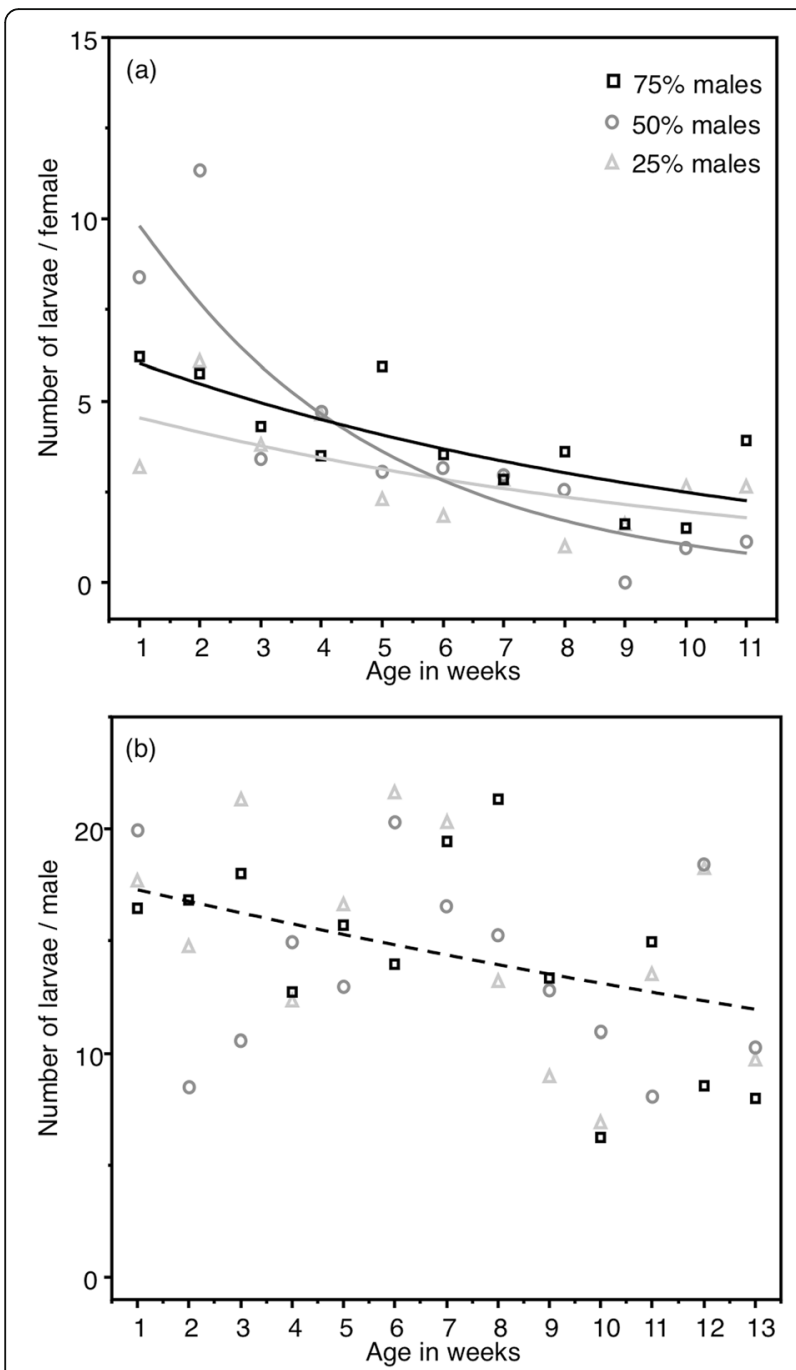

Fig. 2 Age-specific fertility of females (a) and males (b) according to sex-ratio condition. a the tested females were those coming from the experimental tanks. $\mathbf{b}$ the tested females were virgin females mated with males coming from the experimental tanks. Dots are the means (for variation around the means see Additional file 1: Figure S2) and lines are the predictions of the models

again at week 12 (Fig. 4c). In addition, PO activity of males in the 25\%-male sex-ratio condition was overall lower than PO activity of males in the other sex-ratio conditions (Fig. 4c). Total-PO activity only differed between population replicates (Table 3c). As in females, antibacterial activity in the haemolymph of males increased with age (Table 3e, Fig. 4d). However, the size of the zones of inhibition of males exhibiting positive antibacterial activity (reflecting the intensity of this activity) was higher for males in the $50 \%$-male sex-ratio condition than for males in the other sex-ratio conditions (Table 3f, Fig. 4e). Finally, haemocyte counts of males in all sex-ratio conditions varied with time, mainly because of its high value at week 6 (Table 3d, Fig. 4f).

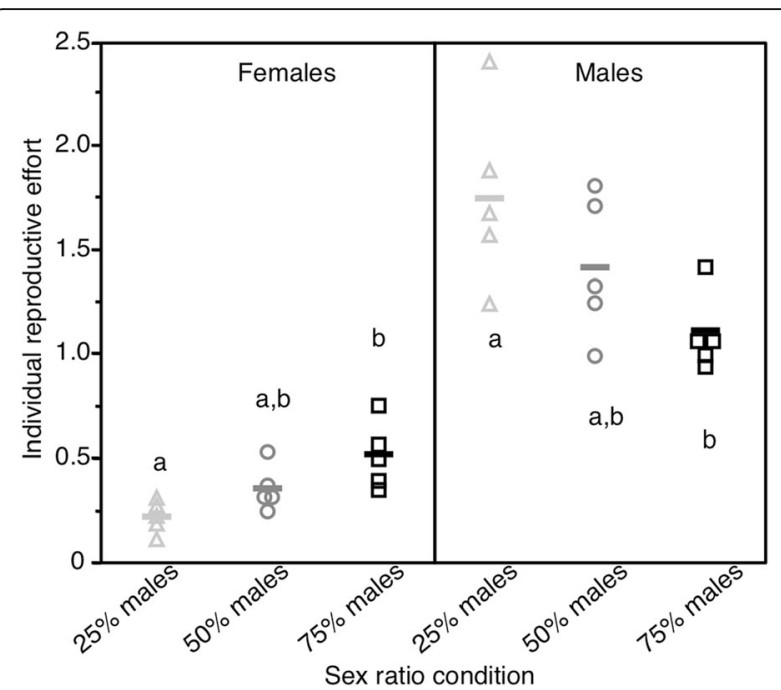

Fig. 3 Estimated mean reproductive effort. Reproductive effort (RE mean number of viable offspring produced per individual and per week of survival in the population) of females (left panel) and males (right panel) according to sex-ratio condition. Lines are means, dots are values of single replicates. Values surrounded by different letters were significantly different after Tukey-Kramer HSD post-hoc test ( $a=0.05$ )

\section{Discussion}

By manipulating the sex-ratio of artificial populations of mealworm beetles, Tenebrio molitor, we successfully affected the reproductive effort of both males and females. Note that for males, our estimations are rather relevant of their reproductive potential effort or maximal reproductive effort because they were tested using young virgin females. As predicted, female biased sex-ratio led females to exhibit a relatively low reproductive effort, whereas males reproductive potential was the highest. By contrast, male biased sex-ratio increased the reproductive effort of females while that of males dropped. Males and females from populations with balanced sex-ratio exhibited intermediate reproductive effort. Interestingly, males and females of the $50 \%$ sex-ratio condition had similar longevity. This absence of divergent patterns of actuarial senescence between males and females may result from a relatively strong investment of males into mating activity rather than into sexual competition. Therefore, like in females, longevity appears to be an important criterion to maximize fitness in males of $T$. molitor.

While varying in their reproductive effort according to sex-ratio condition, females exhibited different patterns of actuarial and reproductive senescence. Females in the $75 \%$-male sex-ratio condition (with the higher reproductive effort) suffered from an increased mortality compared to females in the other conditions. While we did not directly observed the mating behaviour in our experiments, frequent mating events and harassments by males may explain this accelerated mortality and is in line with previous observations from other insect species [34, 63-65]. 
Table 3 Body condition and immune parameters. Mixed linear models or generalized linear model analysing the factors influencing body condition (a), PO activity (b), Total PO activity (c), haemocyte count (d), the proportion of beetles exhibiting antibacterial activity in their haemolymph (e) and the intensity of this antibacterial activity as the size of the zone of inhibition (f) in both females (left) and males (right). Models included sex-ratio condition, Age in weeks (ordinal variable), their interaction, body condition, and replicates as a random factor

\begin{tabular}{|c|c|c|c|c|c|c|c|c|}
\hline & \multicolumn{4}{|c|}{ Females } & \multicolumn{4}{|c|}{ Males } \\
\hline & d.f. & d.f. den. & For $\times 2$ & $P$ & d.f. & d.f. den. & For $\times 2$ & P \\
\hline \multicolumn{9}{|l|}{ A Body condition\# } \\
\hline Age & 3 & 207.9 & 17.14 & $<0.001$ & 3 & 215.1 & 6.64 & $<0.001$ \\
\hline Sex-ratio & 2 & 98.2 & 0.95 & 0.39 & 2 & 89.7 & 0.57 & 0.565 \\
\hline Age $\times$ Sex-ratio & 6 & 207.6 & 0.72 & 0.637 & 6 & 215.1 & 0.23 & 0.964 \\
\hline \multicolumn{9}{|l|}{ B PO activity\# } \\
\hline Age & 3 & 199.8 & 6.76 & $<0.001$ & 3 & 211.9 & 8.20 & $<0.001$ \\
\hline Sex-ratio & 2 & 86.9 & 0.14 & 0.869 & 2 & 103.3 & 3.69 & 0.028 \\
\hline Age $\times$ Sex-ratio & 6 & 198.3 & 0.75 & 0.606 & 6 & 211.3 & 2.21 & 0.043 \\
\hline Body condition & 1 & 208.9 & 6.82 & $<0.001$ & 1 & 222.9 & 2.25 & 0.135 \\
\hline \multicolumn{9}{|l|}{ C Total-PO activity\# } \\
\hline Age & 3 & 189 & 10.90 & $<0.001$ & 3 & 201.8 & 2.49 & 0.061 \\
\hline Sex-ratio & 2 & 189 & 0.21 & 0.807 & 2 & 65.7 & 1.91 & 0.155 \\
\hline Age $\times$ Sex-ratio & 6 & 189 & 0.40 & 0.877 & 6 & 201.2 & 1.25 & 0.282 \\
\hline Body condition & 1 & 189 & 6.10 & 0.014 & 1 & 210.8 & 0.83 & 0.364 \\
\hline \multicolumn{9}{|l|}{ D Haemocyte\# } \\
\hline Age & 3 & 193.8 & 1.28 & 0.281 & 3 & 208 & 6.90 & $<0.001$ \\
\hline Sex ratio & 2 & 11.4 & 0.04 & 0.963 & & & & \\
\hline \multicolumn{9}{|c|}{ E Antibacterial activity (proportion)§ } \\
\hline Age & 3 & & 42.89 & $<0.001$ & 3 & & 12.879 & $<0.001$ \\
\hline Body condition & 1 & & 1.1902 & 0.275 & 1 & & 10.595 & 0.157 \\
\hline Age & & & & & 3 & & 14.391 & 0.002 \\
\hline Sex-ratio & & & & & 2 & & 2.2331 & 0.327 \\
\hline \multicolumn{9}{|c|}{ F Antibacterial activity (intensity)\# ${ }^{1}$} \\
\hline Age & 3 & & 2.27 & 0.088 & & & & \\
\hline Sex-ratio & & & & & 2 & & 4.27 & 0.019 \\
\hline
\end{tabular}

The models presented here are those minimizing the AICc, with $\triangle \mathrm{AICc}>2$. Values where $p \leq 0.05$ are given in bold. \# Linear model, $\S \mathrm{GLM}$, distribution: binomial, link function: Logit, ${ }^{1}$ because a low number of animals showed antimicrobial activity in some conditions (Fig. $4 \mathrm{~b}$, d), data were not available for some replicates, therefore replicates were omitted for these models

Female fertility with time in the $50 \%$ sex-ratio condition contrasted to that of females in the other two sex-ratio conditions. They produced many offspring during the first 2 weeks of their adult life, then fewer to become almost null at 8 weeks onward. As previously reported in both vertebrates and invertebrates $[22,66]$, intense early reproductive activity is associated to earlier reproductive decline. Females in the other sex-ratio conditions ( 25 and $75 \%$ of males) produced relatively fewer offspring when young adults but kept this reproductive effort when becoming older. In the 25\% sex-ratio condition, the low proportion of males may have constrained female access to mating, preventing them to reach their maximal early reproductive potential. Such a low early reproductive investment, possibly accompanied by low male harassment, may have preserved female late reproduction. In the $75 \%$ male sex-ratio condition, high proportion of males was expected to increase interactions between males as well as enhancing mate guarding [38]. This may also have prevented young females to have optimal access to mating, while compensating by a higher probability of mating as they aged. However, female reproduction in this male-biased sex-ratio condition stopped earlier than in the others, because their survival had rapidly declined. All together, the data suggest that the reproductive effort of females in the $75 \%$ male sex-ratio condition was more costly than that of females in the other sex-ratio conditions, constraining them to trade-off their longevity against their reproduction. 

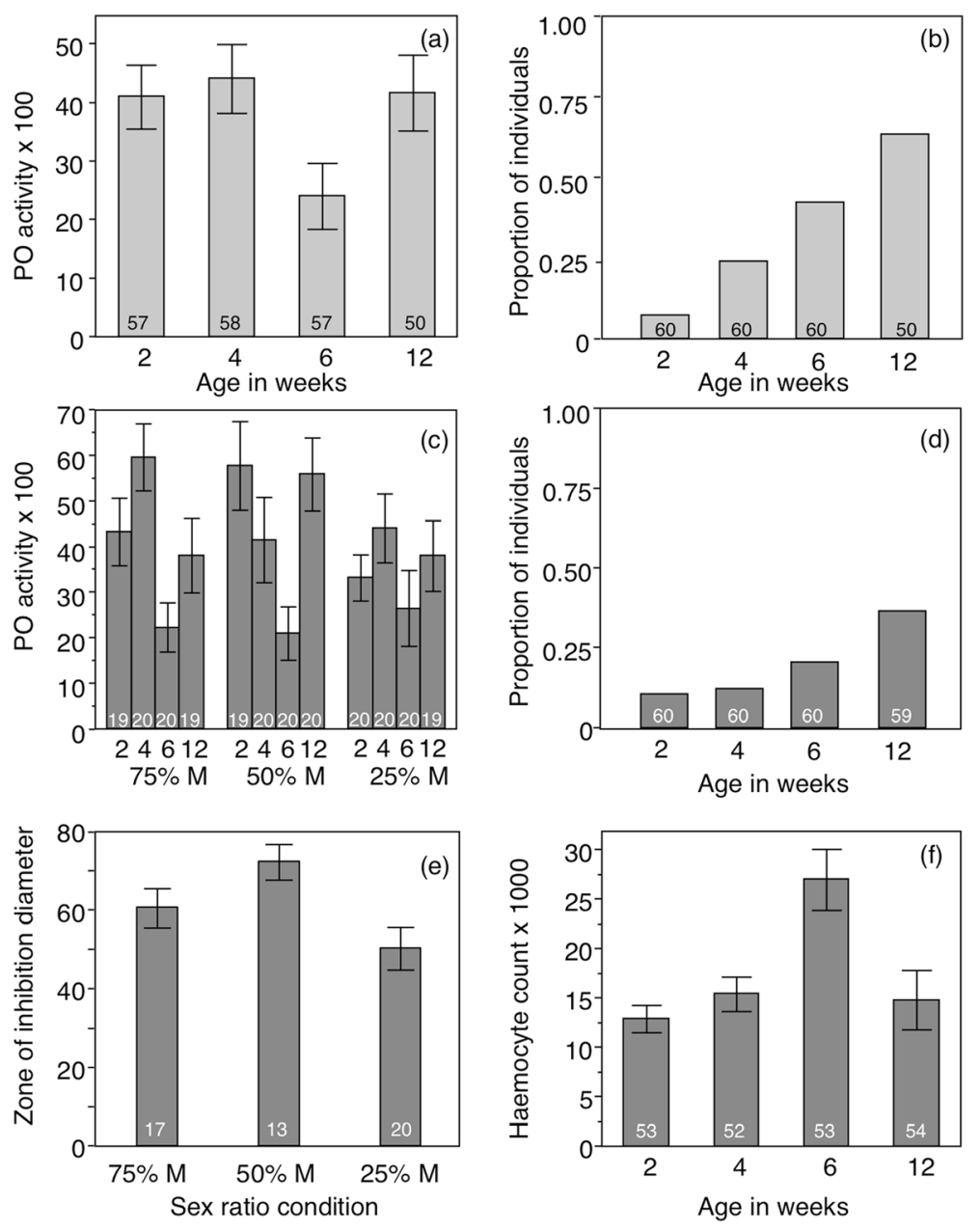

Fig. $4 \mathrm{Immune}$ parameters in females and males according to individual age and/or sex-ratio condition. a females PO activity; b proportion of females exhibiting antibacterial activity; c male PO activity; $\mathbf{d}$ proportion of males exhibiting antibacterial activity; e male intensity of antibacterial activity as the size (in $\mathrm{mm}$ ) of zones of inhibition; $\mathbf{f}$ male haemocyte count. Values are means among replicates $\pm \mathrm{s}$. e. $\mathrm{m}$. Number in the bars are sample size

Despite a more costly reproductive effort, females in the $75 \%$-male sex-ratio condition did not exhibit any further functional decline compare to females in the other sex-ratio conditions. While body condition declined with female age, such a decline was similar in all sex-ratio conditions. Similarly, changes in female immune activity were never influenced by the sex-ratio. While haemocyte counts remained constant with female age, antibacterial activity increased. Similar results were reported in the bumblebee, Bombus terrestris [53]. With age, the probability of having been exposed to microbes increases. This may explain the higher proportion of older individuals exhibiting induced antibacterial activity in their haemolymph, as insects can produce prophylactic long lasting antibacterial responses after a single bacterial challenge $[67,68]$. PO activity declined at week 6 in females, which is consistent with the beginning of senescence, when reproduction started to end but survival is still relatively high (Fig. 1, see Additional file 1: Figure S2). PO activity increased again at week 12, among the rare surviving individuals (Fig. 1). This higher late PO-activity may have two non-exclusive explanations. On the one hand, it may result from selection where individuals with the best somatic protection, involving high $\mathrm{PO}$-activity, survived longer than those having poorer ones. On the other hand, high levels of PO activity at week 12 could also result from a deregulation of the host inflammatory response $[69,70]$. The impact of female reproductive effort on immunity seems limited, at least on the constitutive base levels of the immune parameters we have measured. We cannot exclude that female ability to produce an immune response upon challenge or others non-measured immune parameters could be affected. Nonetheless, our results show that increasing the reproductive effort of $T$. molitor females affected demographic senescence, mainly through longevity 
reduction, but with apparently limited effect on immune senescence.

Changes in the reproductive effort (reproductive potential) of males through the manipulation of the sexratio also affected their survival. It is often assumed that most of the cost of reproduction in males involves sexual pre-copulatory competition. Thus males in the $75 \%$-male sex-ratio condition could have been expected to engage in strong and costly intra-sexual competition for females, resulting in low reproductive success and shorter longevity compared to the other sex-ratio conditions, as previously shown in vertebrates $[11,71,72]$ and invertebrates [34]. However, although male fertility slightly declined with age, it was not affected by the experimental sex-ratio condition, suggesting that males exhibited similar patterns of reproductive senescence, independently of their reproductive effort. Male reproductive senescence might also be revealed by the production of lower quality offspring with age [73], which was not tested in this study. In addition, males from the $75 \%$-sex-ratio condition showed longer longevity. This phenomenon may have two main explanations, consistent with predictions linked to the peculiar mating behaviour of $T$. molitor. On the one hand, competition for females in that sexratio condition was not very strong or costly. Under high risk of sexual competition, male reproductive success may depend on their investment into pre-copulatory (e.g., courtship and aggressive behaviours with other males) and/or post-copulatory (e.g., mate guarding) behaviours to limit sperm competition [74]. So far, T. molitor males were never reported to engage in physical contest either before or after copulation [38] and current evidence suggests that male-male competition is unlikely to bear strong costs [38, 41, 75, 76]. Our experimental design nevertheless did not allowed us to verify these assumptions. On the other hand, each mating event is costly for $T$. molitor males, because nutrient-rich spermatophores are transferred to females in addition to the sperm [42]. Since, on average, males in the $75 \%$-male sex-ratio condition may have copulated less frequently than males in the other sex-ratio conditions, they may have saved resources that contributed to their longer survival. By contrast, males from the $25 \%$-male sex-ratio condition likely performed more mating events than males in the other sex-ratio conditions, but this was apparently not costly enough to significantly impair their longevity compared to the 50\%-male sex-ratio condition.

Our results suggest that males in this 25\%-male sexratio condition had paid a functional cost for their higher reproductive effort, especially in terms of immunity. Indeed, males in the 25\%-male sex-ratio condition showed a reduced immune activity possibly resulting from their higher reproductive effort. First, males in the 25\%-male sex-ratio condition had reduced PO activity despite having a similar concentration of total phenoloxidase enzymes in their haemolymph than males of the other sex-ratio conditions. This lower PO activity was constant over time and contrasted with that of males of the two other sex-ratio conditions, for which the temporal pattern of PO activity resembled that of females (high levels in early weeks, decline in week 6, and reincrease in week 12). Since mating activity is known to transiently reduce $\mathrm{PO}$ activity in $T$. molitor [52], such a down regulation of the PO activity in males of the $25 \%$ male sex-ratio condition might be reflecting their higher mating activities. Higher secretion of juvenile hormone might be involved in mediating mating-induced PO activity depression [52], which could contribute to reduce longevity [77]. Juvenile hormone also prevents the release of cytotoxic substances by active PO enzymes that could reduce insect longevity by self-damaging host tissues and organs [47, 49-51, 78]. These combined effects may have contributed to the observed absence of difference between the survival of males in the $25 \%$ and the $50 \%$-male sex-ratio conditions. Second, as observed for females, the proportion of males exhibiting positive antibacterial activity in their haemolymph increased with age in all the sex-ratio conditions. As stated earlier, this was expected as the probability of having being challenged by opportunistic microbes increases with age. However, the size of the zones of inhibition observed from the haemolymph of males in the $25 \%$-male sexratio condition was significantly smaller than that of males in the other sex-ratio conditions, suggesting that males in the $25 \%$-male sex-ratio condition produced less antibacterial peptides than the other males. As mating activity, through the production and transfer of spermatophores, is particularly resource-demanding for males in terms of protein content [42], the higher mating activity of males in the $25 \%$-male sex-ratio condition could have depleted the necessary protein resource to produce as much antibacterial peptides as in the other sex-ratio conditions. Mating may mediate such a tradeoff through juvenile hormone secretion, which functions to switch on physiological processes associated with gametogenesis and spermatophore production [79].

\section{Conclusions}

Manipulating sex-ratio of artificial populations of $T$. molitor had important impacts on reproductive effort of females and males, but resulted in contrasting sexspecific trade-offs on demographic and immune traits. Increasing female reproductive effort did not affect immunity but strongly reduced longevity. Not surprisingly, females may then maximize fitness by moderate early investment into reproduction and longevity. While decreasing male reproductive effort enhanced longevity, increasing it impairs immunity. Males may therefore 
favour reproduction at the expense of their immunity when given the opportunity to increase their reproductive effort. This is in line with the Bateman's principle applied to immunity, where males gain fitness by increasing reproductive effort at the expense of immunity [80]. It is also consistent with the disposable soma theory of ageing, as reproduction compromises somatic protection $[3,4]$. Nevertheless, our results also suggest that sexual competition in $T$. molitor is not a strong modulator of the male reproductive strategy towards early mating opportunities [81]. Basically, like in females, most of the cost of reproduction in males results from multiple copulations. This thus contrasts with the hypothesis that males should gain fitness by increasing mating success by investing in sexual competition at the expense of longevity [82, 83]. Since longevity is a key life history trait for both males and females of T. molitor, sex-specific patterns of actuarial senescence are not expected to evolve in this species. Accordingly, males and females showed similar patterns of survival with age in populations with balanced sex-ratio. Our results may further indirectly suggest that divergent actuarial senescence between males and females should evolve in species in which males strongly invest into sexual competition $[11,22]$.

\section{Supplementary information}

Supplementary information accompanies this paper at https://doi.org/10 1186/s12862-020-1586-X

Additional file 1: Table S1. AICC values for the models presented in Tables 2 and 3. The models in bold are those presented in the tables. Figure S1. Male and female age specific mortality rate according to time for each sex-ratio condition. Arrow indicate the time when the values of $50 \%$ of mortality rate is reached for the first time and dashed lines indicate $50 \%$ of the population is dead. Values are means among replicates \pm s. e. m.

Figure S2. Details of variation in fertility in females (A) and males (B) Values are means among replicates \pm s. e. m. Figure S3. Physiological parameters: females in bright grey and males in black. Body condition, PO activity according to time, Total-PO activity according to time and sex-ratio condition, Haemocyte count, Proportion of individuals producing antibacterial activity, Diameter of inhibition zone according to time and sex-ratio condition. Values are means among replicates $\pm \mathrm{s}$. e. $\mathrm{m}$.

\section{Abbreviations}

AICc: Corrected Akaike Information Criterion; ANOVA: Analysis of Variance; I: Number of larvae; ML: Mean Lifespan; PBS: Phosphate Buffer Saline; PO: Phenoloxidase; ProPO: ProPhenoloxidase; $r$ : Replicate; RE: Reproductive Effort; REML: REstricted Maximum Likelihood

\section{Acknowledgements}

We thank G. Sorci for critical comments on the manuscript, C. Sabarly, A. Salis and M. Teixeira Brandao for technical assistance.

\section{Authors' contributions}

CJ, TR and YM conceived the ideas and designed methodology, CJ, MC and YM collected the data, CJ, TR and YM analysed the data, CJ, TR and YM led the writing of the manuscript. All authors contributed critically to the drafts and gave final approval for publication.

\section{Funding}

This study was funded by the CNRS and a grant from the ANR (ANR-14CE02-0009). Funding agencies contributed strictly financially to the performed research.

Availability of data and materials

All data files will be available from the Dryad Digital database at https://doi.org/ 10.5061/dryad.cvdncjt11.

Ethics approval and consent to participate

Not applicable.

\section{Consent for publication}

Not applicable.

\section{Competing interests}

The authors declare that they have no competing interests.

Received: 13 November 2019 Accepted: 24 January 2020

Published online: 03 February 2020

\section{References}

1. Stearns SC. The evolution of life histories. Oxford: Oxford University Press; 1992.

2. Hughes KA, Reynolds RM. Evolutionary and mechanistic theories of aging. Annu Rev Entomol. 2005;50:421-45

3. Williams GC. Natural selection, the costs of reproduction, and a refinement of Lack's principle. Am Nat. 1966;100:687-90.

4. Kirkwood TB, Rose MR. Evolution of senescence: late survival sacrificed for reproduction. Philos Trans R Soc Lond Ser B Biol Sci. 1991;332:15-24.

5. Jones OR, Scheuerlein A, Salguero-Gómez R, Camarda CG, Schaible R, Casper BB, et al. Diversity of ageing across the tree of life. Nature. 2014;505: 169-73.

6. Jones OR, Gaillard J-M, Tuljapurkar S, Alho JS, Armitage KB, Becker PH, et al. Senescence rates are determined by ranking on the fast-slow life-history continuum. Ecol Lett. 2008;11:664-73.

7. Rodríguez-Muñoz R, Boonekamp JJ, Liu XP, Skicko I, Fisher DN, Hopwood P, et al. Testing the effect of early-life reproductive effort on age-related decline in a wild insect. Evolution. 2019;73:317-28.

8. van Noordwijk AJ, de Jong G. Acquisition and allocation of resources: their influence on variation in life history tactics. Am Nat. 1986;128:137-42.

9. Bleu J, Gamelon M, Sæther B-E. Reproductive costs in terrestrial male vertebrates: insights from bird studies. Proc R Soc B Biol Sci. 2016;283: 20152600.

10. Hamel S, Gaillard J-M, Yoccoz NG, Loison A, Bonenfant C, Descamps S. Fitness costs of reproduction depend on life speed: empirical evidence from mammalian populations. Ecol Lett. 2010;13:915-35.

11. Lemaitre J-F, Gaillard J-M, Pemberton JM, Clutton-Brock TH, Nussey DH. Early life expenditure in sexual competition is associated with increased reproductive senescence in male red deer. Proc R Soc B Biol Sci. 2014;281: 20140792.

12. Tidière M, Gaillard J-M, Müller DWH, Lackey LB, Gimenez O, Clauss M, et al. Does sexual selection shape sex differences in longevity and senescence patterns across vertebrates? A review and new insights from captive ruminants. Evolution. 2015;69:3123-40.

13. Walker WF. Sperm utilization strategies in nonsocial insects. Am Nat. 1980; 115:780-99.

14. Dewsbury DA. Ejaculate cost and male choice. Am Nat. 1982:119:601-10.

15. Andersson MB. Sexual selection. Princeton: Princeton University Press; 1994.

16. Arnqvist G, Rowe L. Sexual conflict. Princeton: Princeton University Press; 2005.

17. Hosken DJ. Sex and death: microevolutionary trade-offs between reproductive and immune investment in dung flies. Curr Biol. 2001;11:R379-80.

18. Stockley P. Sexual conflict resulting from adaptations to sperm competition Trends Ecol Evol. 1997:12:154-9.

19. Jennions MD, Petrie M. Why do females mate multiply? A review of the genetic benefits. Biol Rev Camb Philos Soc. 2000;75:21-64.

20. Harshman LG, Zera AJ. The cost of reproduction: the devil in the details. Trends Ecol Evol. 2007:22:80-6.

21. Fortin M, Meunier J, Laverré T, Souty-Grosset C, Richard F-J. Joint effects of group sex-ratio and Wolbachia infection on female reproductive success in the terrestrial isopod Armadillidium vulgare. BMC Evol Biol. 2019;19:65. 
22. Lemaître J-F, Berger V, Bonenfant C, Douhard M, Gamelon M, Plard F, et al. Early-late life trade-offs and the evolution of ageing in the wild. Proc R Soc B Biol Sci. 2015;282:20150209.

23. DeVeale B, Brummel T, Seroude L. Immunity and aging: the enemy within? Aging Cell. 2004;3:195-208.

24. Shanley DP, Aw D, Manley NR, Palmer DB. An evolutionary perspective on the mechanisms of immunosenescence. Trends Immunol. 2009;30:374-81.

25. Norris K, Evans MR. Ecological immunology: life history trade-offs and immune defense in birds. Behav Ecol. 2000;11:19-26.

26. Owens IPF, Wilson K, Owens IPF, Wilson K. Immunocompetence: a neglected life history trait or conspicuous red herring? Trends Ecol Evol. 1999;14:170-2.

27. Zera AJ, Harshman LG. The physiology of life history trade-offs in animals. Annu Rev Ecol Syst. 2001;32:95-126.

28. Fedorka KM, Zuk M, Mousseau TA. Immune suppression and the cost of reproduction in the ground cricket, Allonemobius socius. Evolution. 2004;58: 2478-85.

29. Simmons LW, Roberts B. Bacterial immunity traded for sperm viability in male crickets. Science. 2005;309:2031.

30. French SS, DeNardo DF, Moore MC. Trade-offs between the reproductive and immune systems: facultative responses to resources or obligate responses to reproduction? Am Nat. 2007;170:79-89.

31. Drummond-Barbosa D, Spradling AC. Stem cells and their progeny respond to nutritional changes during Drosophila oogenesis. Dev Biol. 2001;231:265-78.

32. Greenman CG, Martin LB, Hau M. Reproductive state, but not testosterone, reduces immune function in male house sparrows (Passer domesticus). Physiol Biochem Zool. 2005;78:60-8.

33. Schwenke RA, Lazzaro BP, Wolfner MF. Reproduction-immunity trade-offs in insects. Annu Rev Entomol. 2016;61:239-56.

34. Rodríguez-Muñoz R, Boonekamp JJ, Fisher D, Hopwood P, Tregenza T. Slower senescence in a wild insect population in years with a more femalebiased sex ratio. Proc Biol Sci. 2019;286:20190286.

35. Tompkins EM, Anderson DJ. Sex-specific patterns of senescence in Nazca boobies linked to mating system. J Anim Ecol. 2019;88:986-1000.

36. Clutton-Brock TH, Parker GA. Potential reproductive rates and the operation of sexual selection. Q Rev Biol. 1992;67:437-56.

37. Klug H, Heuschele J, Jennions MD, Kokko H. The mismeasurement of sexual selection. J Evol Biol. 2010;23:447-62.

38. Carazo P, Font E, Alfthan B. Chemosensory assessment of sperm competition levels and the evolution of internal spermatophore guarding. Proc Biol Sci. 2007;274:261-7.

39. Gage MJG, Baker RR. Ejaculate size varies with socio-sexual situation in an insect. Ecol Entomol. 1991;16:331-7.

40. Gadzama NM, Happ GM. The structure and evacuation of the spermatophore of Tenebrio molitor L. (Coleoptera: Tenebrionidae). Tissue Cell. 1974;6:95-108.

41. Drnevich JM. Number of mating males and mating interval affect last-male sperm precedence in Tenebrio molitor L. Anim Behav. 2003;66:349-57.

42. Carver FJ, Gilman JL, Hurd H. Spermatophore production and spermatheca content in Tenebrio molitor infected with Hymenolepis diminuta. J Insect Physiol. 1999;45:565-9.

43. Dick J. Oviposition in certain Coleoptera. Ann Appl Biol. 1937;24:762-96.

44. Drnevich JM, Papke RS, Rauser $C L$, Rutowski RL. Material benefits from multiple mating in female mealworm beetles (Tenebrio molitor L.). J Insect Behav. 2001:14:215-30.

45. Vigneron A, Jehan C, Rigaud T, Moret Y. Immune defenses of a beneficial pest: the mealworm beetle, Tenebrio molitor. Front Physiol. 2019;10. https:// doi.org/10.3389/fphys.2019.00138.

46. Nappi AJ, Ottaviani E. Cytotoxicity and cytotoxic molecules in invertebrates. Bioessays. 2000;22:469-80.

47. Sadd BM, Siva-Jothy MT. Self-harm caused by an insect's innate immunity, Proc Biol Sci. 2006:273:2571-4.

48. Pursall ER, Rolff J. Immune responses accelerate ageing: proof-of-principle in an insect model. PLoS One. 2011:6:e19972.

49. Daukšte J, Kivleniece I, Krama T, Rantala MRJ, Krams IA. Senescence in immune priming and attractiveness in a beetle. J Evol Biol. 2012;25:1298-304.

50. Krams I, Daukšte J, Kivleniece I, Kaasik A, Krama T, Freeberg TM, et al. Tradeoff between cellular immunity and life span in mealworm beetles Tenebrio molitor. Curr Zool. 2013;59:340-6.

51. Khan I, Agashe D, Rolff J. Early-life inflammation, immune response and ageing. Proc R Soc B Biol Sci. 2017;284:20170125.
52. Rolff J, Siva-Jothy MT. Copulation corrupts immunity: a mechanism for a cost of mating in insects. PNAS. 2002;99:9916-8.

53. Moret $Y$, Schmid-Hempel P. Immune responses of bumblebee workers as a function of individual and colony age: senescence versus plastic adjustment of the immune function. Oikos. 2009;118:371-8.

54. Khan I, Prakash A, Agashe D. Immunosenescence and the ability to survive bacterial infection in the red flour beetle Tribolium castaneum. J Anim Ecol. 2016;85:291-301.

55. Moret $Y$, Schmid-Hempel P. Survival for immunity: the price of immune system activation for bumblebee workers. Science. 2000;290:1166-8.

56. Licastro F, Candore G, Lio D, Porcellini E, Colonna-Romano G, Franceschi C, et al. Innate immunity and inflammation in ageing: a key for understanding agerelated diseases. Immun Ageing. 2005. https://doi.org/10.1186/1742-4933-2-8.

57. Shenk MK. Fertility and fecundity. In: The international encyclopedia of human sexuality. New York City: American Cancer Society; 2015. p. 369-426. https://doi.org/10.1002/9781118896877.wbiehs153.

58. Moret $Y$. "Trans-generational immune priming": specific enhancement of the antimicrobial immune response in the mealworm beetle, Tenebrio molitor. Proc Biol Sci. 2006:273:1399-405.

59. Zanchi C, Troussard J-P, Martinaud G, Moreau J, Moret Y. Differential expression and costs between maternally and paternally derived immune priming for offspring in an insect. J Anim Ecol. 2011;80:1174-83.

60. Norušis MJ. IBM SPSS Statistics 19 statistical procedures companion. Upper Saddle River: Prentice Hall; 2012. https://trove.nla.gov.au/version/173310980. Accessed 31 Oct 2019

61. Burnham KP, Anderson DR. Multimodel inference: understanding AIC and BIC in model selection. Sociol Methods Res. 2004;33:261-304.

62. Jehan C, Chogne M, Rigaud T, Moret Y. Data from: sex-specific patterns of senescence in artificial insect populations varying in sex-ratio to manipulate reproductive effort. Dryad Digital Repository. 2020. https://doi.org/10.5061/ dryad.cvdncjt11.

63. Adler Ml, Bonduriansky R. The dissimilar costs of love and war: age-specific mortality as a function of the operational sex ratio. J Evol Biol. 2011;24: 1169-77.

64. Archer CR, Zajitschek F, Sakaluk SK, Royle NJ, Hunt J. Sexual selection affects the evolution of lifespan and ageing in the decorated cricket Gryllodes Sigillatus. Evolution. 2012;66:3088-100.

65. Tatar M, Carey JR, Vaupel JW. Long-term cost of reproduction with and without accelerated senescence in callosobruchus maculatus: analysis of age-specific mortality. Evolution. 1993;47:1302-12.

66. Creighton JC, Heflin ND, Belk MC. Cost of reproduction, resource quality, and terminal investment in a burying beetle. Am Nat. 2009;174:673-84.

67. Haine ER, Pollitt LC, Moret Y, Siva-Jothy MT, Rolff J. Temporal patterns in immune responses to a range of microbial insults (Tenebrio molitor). J Insect Physiol. 2008:54:1090-7.

68. Dhinaut J, Chogne M, Moret $Y$. Immune priming specificity within and across generations reveals the range of pathogens affecting evolution of immunity in an insect. J Anim Ecol. 2018;87:448-63.

69. Scherfer C, Tang H, Kambris Z, Lhocine N, Hashimoto C, Lemaitre B. Drosophila Serpin-28D regulates hemolymph phenoloxidase activity and adult pigmentation. Dev Biol. 2008;323:189-96.

70. Labaude S, Moret Y, Cézilly F, Reuland C, Rigaud T. Variation in the immune state of Gammarus pulex (Crustacea, Amphipoda) according to temperature: are extreme temperatures a stress? Dev Comp Immunol. 2017;76:25-33.

71. Promislow DEL. Costs of sexual selection in natural populations of mammals. Proc R Soc Lond Ser B Biol Sci. 1992;247:203-10.

72. Clutton-Brock TH, Isvaran K. Sex differences in ageing in natural populations of vertebrates. Proc Biol Sci. 2007;274:3097-104.

73. Carazo P, Molina-Vila P, Font E. Male reproductive senescence as a potential source of sexual conflict in a beetle. Behav Ecol. 2011;22:192-8.

74. Font E, Desfilis E. Chapter 3 - courtship, mating, and sex pheromones in the mealworm beetle (Tenebrio molitor). In: Ploger BJ, Yasukawa K, editors. Exploring animal behavior in laboratory and field. San Diego: Academic Press; 2003. p. 43-58. https://doi.org/10.1016/B978-012558330-5/50005-4

75. Happ GM. Multiple sex pheromones of the mealworm beetle, Tenebrio molitor L. Nature. 1969;222:180.

76. Drnevich JM, Hayes EF, Rutowski RL. Sperm precedence, mating interval, and a novel mechanism of paternity bias in a beetle (Tenebrio molitor L.). Behav Ecol Sociobiol. 2000;48:447-51.

77. Herman WS, Tatar M. Juvenile hormone regulation of longevity in the migratory monarch butterfly. Proc Biol Sci. 2001;268:2509-14. 
78. Zhao P, Lu Z, Strand MR, Jiang H. Antiviral, anti-parasitic, and cytotoxic effects of 5,6-dihydroxyindole (DHI), a reactive compound generated by phenoloxidase during insect immune response. Insect Biochem Mol Biol. 2011:41:645-52.

79. Wigglesworth VB. The principles of insect physiology. Netherlands: Springer; 1972. https://www.springer.com/la/book/9780412246609. Accessed 4 Apr 2019

80. Rolff J. Bateman's principle and immunity. Proc Biol Sci. 2002;269:867-72.

81. Bonduriansky R, Maklakov A, Zajitschek F, Brooks R. Sexual selection, sexual conflict and the evolution of ageing and life span. Funct Ecol. 2008;22:443-53

82. Vinogradov AE. Male reproductive strategy and decreased longevity. Acta Biotheor. 1998;46:157-60

83. Carranza J, Pérez-Barbería FJ. Sexual selection and senescence: male sizedimorphic ungulates evolved relatively smaller molars than females. Am Nat. 2007;170:370-80.

\section{Publisher's Note}

Springer Nature remains neutral with regard to jurisdictional claims in published maps and institutional affiliations.

Ready to submit your research? Choose BMC and benefit from:

- fast, convenient online submission

- thorough peer review by experienced researchers in your field

- rapid publication on acceptance

- support for research data, including large and complex data types

- gold Open Access which fosters wider collaboration and increased citations

- maximum visibility for your research: over $100 \mathrm{M}$ website views per year

At BMC, research is always in progress.

Learn more biomedcentral.com/submissions 